\title{
EVALUATION OF CRITICAL EXPERIMENTS IN THE UNIVERSITY OF WISCONSIN NUCLEAR REACTOR (UWNR) WITH UNCERTAINTY QUANTIFICATION
}

\author{
Young-Hui Park ${ }^{1}$, Ye Cheng ${ }^{2}$, Rabab Elzohery ${ }^{2}$, \\ Paul P.H. Wilson ${ }^{1}$, Jeremy A. Roberts ${ }^{2}$, and Mark D. DeHart ${ }^{3}$ \\ ${ }^{1}$ Computational Nuclear Engineering Research Group, \\ Department of Engineering Physics, University of Wisconsin-Madison \\ 1415 Engineering Drive, Madison, WI 53705, US \\ ${ }^{2}$ Computation and Optimization for Reactor Physics Simulation Group, \\ Department of Mechanical and Nuclear Engineering, Kansas State University \\ 3002 Rathbone Hall, Manhattan, KS 66506, US \\ ${ }^{3}$ Reactor Physics Analysis and Design Department, \\ Idaho National Laboratory \\ Idaho Falls, ID 83415, US \\ ypark234@wisc.edu, yc4v7@ksu.edu, rababelzohery@ksu.edu, \\ paul.wilson@wisc.edu, jaroberts@ksu.edu, mark.dehart@inl.gov
}

\begin{abstract}
An improved computational model of the University of Wisconsin Nuclear Reactor (UWNR) was developed to support the benchmark evaluation of recent data acquired during an experimental campaign conducted at UWNR. Previous efforts led to a scripted UWNR model for automated generation of MCNP6 and Serpent inputs. This capability was extended to SCALE/KENO. All three tools were used to evaluate a variety of zero-power, fresh-critical configurations, and the results agreed well. The MCNP6 model was extended to support shuffling the core configuration, which allows the modeling of burnup for evaluation of depleted critical configurations. The MCNP6 model successfully predicts core reactivity over time, after accounting for the initial reactivity bias. The inclusion of SCALE/KENO input generation enables sensitivity and uncertainty analyses using the TSUNAMI and Sampler modules of SCALE. A preliminary uncertainty analysis was performed with TSUNAMI for nuclear data uncertainties while direct perturbation calculations were performed using MCNP6 for geometry and material uncertainties, which helped to identify model parameters with the largest effect on the eigenvalue. A transient UWNR transport Model in Mammoth/Rattlesnake is under development to simulate the transient experiments. The existing MCNP6 and Serpent models are used to provide the CAD file for meshing and homogenized cross-sections. In conclusion, the evaluation of UWNR benchmark data provides increased confidence in various states of the UWNR computational model and will provide a unique model for use by other analysts.
\end{abstract}

KEYWORDS: Benchmark, critical configuration, reaction rate measurement, uncertainty quantification, transient experiment 


\section{INTRODUCTION}

The University of Wisconsin Nuclear Reactor (UWNR) located in Madison, Wisconsin is a 1 megawatt (MW) TRIGA ${ }^{\circledR}$ (Training, Research, Isotopes, General Atomics) reactor converted from MTR (Materials Testing Reactor). Efforts have been made to improve the computational model of the reactor as a part of a NEUP (Nuclear Energy University Program) NEAMS (Nuclear Energy Advanced Modeling and Simulation) project on evaluation of experimental data measured in UWNR[1,2]. These improvements include (1) improved data provenance, (2) build consistency from automatic input generation using Python scripts, (3) human readability and transparency of model changes, (4) configuration management by version control, and (5) modularity for alternate representations. Previous efforts have added the capability of generating inputs for Serpent[3] as an alternate representation of the existing MCNP6[4] model, providing cross-code validation and in-depth burnup analysis[5]. The present work adds another layer on the modularity of the UWNR computational model, leading to an increased confidence on validation of the model as well as providing additional tools for more in-depth analysis. With the addition of SCALE/KENO[6] to the model, detailed sensitivity and uncertainty analyses have been facilitated by utilizing TSUNAMI and Sampler, submodules of SCALE. Meanwhile, the MCNP6 model was extended with a capability to track core depletion across different core configurations. With this feature, core depletion can be simulated from the initial J21-R14 core through core shuffles to the current K21-R6 core (see Figure 1 for configuration details), allowing comparison with reactor operation logs as another way of validating the model. Overall model bias can be established by simulating the approachto-critical experiment which is a set of criticality measurements at the time of initial core loading. With this bias adjusted over the lifetime of the reactor, core depletion simulation results can be better compared with reactor operation log.

\section{MODEL DESCRIPTIONS}

The computational model of the UWNR is built using raw data including dimensions of major reactor components and as-built fuel compositions. The model is scripted in Python to automate generation of input files for MCNP6, Serpent, and KENO and to ensure the consistency of input files generated.

\subsection{Model Geometry}

The reactor core is located near the bottom of a $8.38 \mathrm{~m}$ deep pool surrounded by a concrete shield. Several beam ports and a thermal column extend from the outer surface of the concrete shield to the edge of the core. The core is composed of fuel bundles and graphite reflectors placed on a 9by- 7 grid plate. Blade-shaped control elements divide the core into 3 regions. Each of the 21 fuel bundles is composed of 4 fuel elements in a $2 \times 2$ grid, except one near the core center that contains the transient rod in place of one fuel element. Each fuel element consists of cylindrical fuel meat with a central zirconium rod. The fuel meat is axially divided into three "slugs" of roughly equal height. To the top and bottom of the fuel are cylindrical, graphite reflectors. The fuel meat and graphite are enclosed in stainless steel cladding and capped with stainless steel end fittings.

The core was originally placed in the J21-R14 configuration, which consists of 21 fuel bundles and 14 graphite reflectors, as shown in Figure 1(a). Start-up tests indicated a lower reactivity than 


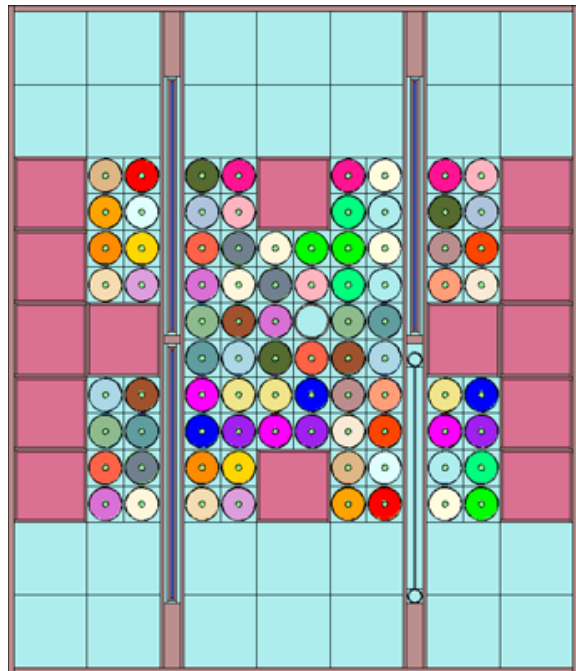

(a) J21-R14 core configuration

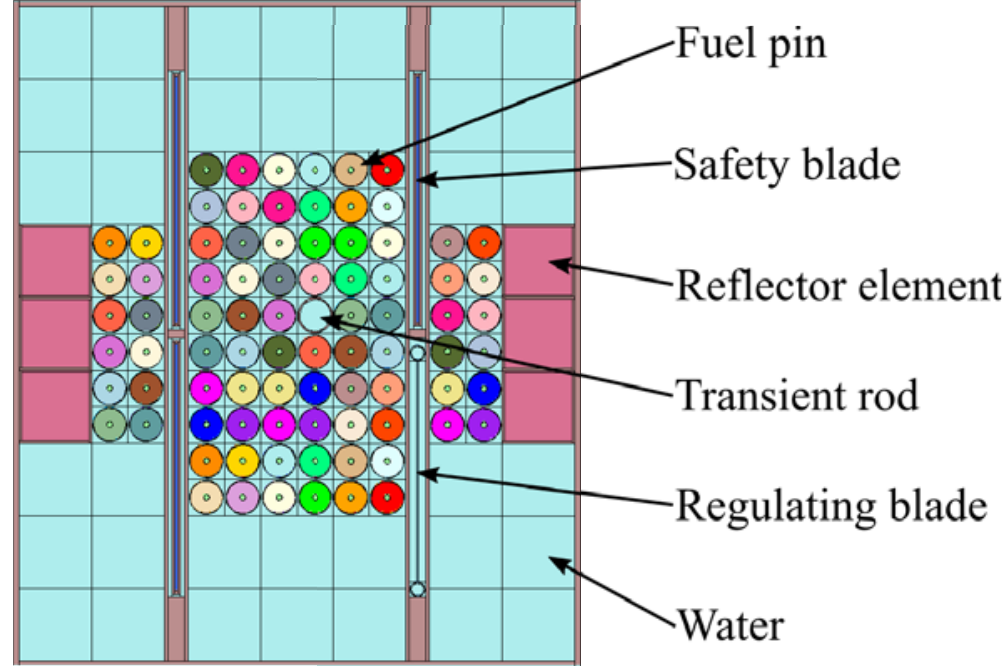

(b) K21-R06 core configuration

\section{Figure 1: MCNP6-plotted geometry of UWNR core. Fuel pins are represented in various colors as they have different material numbers for burnup simulation.}

predicted. Further analysis led to the development of the K21-R06 configuration shown in Figure 1(b). All fresh critical data were generated with the J-core configuration. Depleted critical data and the recent experiments are based on the correct K-core configuration.

\subsection{Material Composition}

The UWNR was completely refueled in 2009 with low enriched uranium (LEU) 30/20 fuel (30 wt \% $\mathrm{U}$ enriched to $20 \%$ in U-235). The fuel meat is a homogeneous mixture of $\mathrm{UZrH}_{1.6}$ with Er poison ( 0.9 w/o). Compositions for each fuel slug were provided by the manufacturer and, therefore, support the detailed modeling of fresh critical configurations and the evolution of materials for depleted critical configuration. More detail on these compositions can be found elsewhere[2].

The reactor is controlled by elements of three types, each with a different composition and purpose. The cylindrical transient rod is made of boron carbide with a stainless steel cladding inside an aluminum guide tube. The three safety blades are made of boral (35 wt \% boron carbide, $65 \mathrm{wt} \%$ aluminum) with an aluminum cladding. Finally, the regulating blade is a stainless steel sheet welded to stainless steel tubes on vertical edges.

\section{MODEL VALIDATION}

Preliminary verification and validation $(\mathrm{V} \& \mathrm{~V})$ of the model was performed via comparison of MCNP6 and Serpent results for a set of fresh critical configurations [2]. Here, work to support further $\mathrm{V} \& \mathrm{~V}$ is presented and includes the addition of KENO results for the fresh configurations and the initial development of a depletion sequence and its comparison to operational data. 


\subsection{Approach to Critical Experiment on Fresh Critical Configuration}

As part of refueling, a sequence of critical experiments collectively known as the approach-tocritical (ATC) experiments was performed. Fuel bundles were added to an empty core, one at a time. Upon initial criticality, critical control element bank heights were determined and recorded for each additional fuel bundle. Following each fuel-bundle addition, single reflector worth measurements were made at multiple positions, and additional reflector elements were inserted. In total, the experiments included 21 unique, fresh critical configurations, where, e.g., configuration J21-R01-a2 has 21 fuel bundles and one reflector in the peripheral, A2 position.

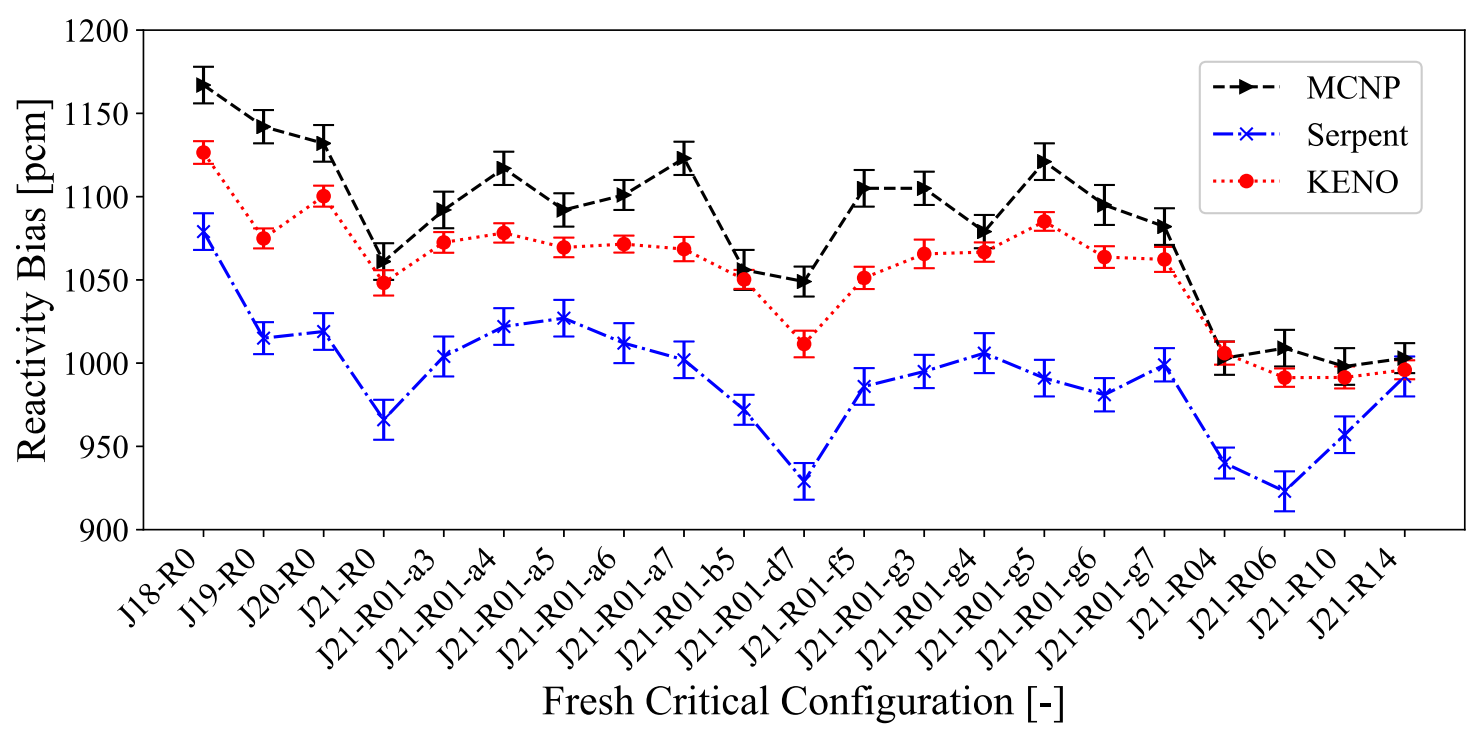

Figure 2: Comparison of MCNP6, Serpent, and KENO results for ATC experiments.

Simulations were performed using MCNP6, Serpent, and KENO for each ATC configuration using slug-averaged fuel compositions. Departures from criticality represent reactivity biases and are shown for each code and configuration in Figure 2. For the simulations, $10^{6} / 10^{5} / 10^{6}$ histories per cycle, 20/200/20 inactive cycles, and 80/500/230 active cycles were used with MCNP6, Serpent, and KENO, respectively.

The KENO results shown in Figure 2 provide increased confidence in the UWNR computational model. Specifically, the KENO results lie between MCNP6 and Serpent results, and exhibit the same systematic bias over all configurations. The mean reactivity biases observed are $1071 \pm 46$ pcm, $982 \pm 34$ pcm, and $1044 \pm 35$ pcm for MCNP6, Serpent, and KENO.

\subsection{Depletion Comparison with Reactor Operation Log}

Detailed records of core burnup, critical bank heights, and excess core reactivity have been made since the 2009 fuel conversion and provide one source of data for validation of the model for depleted fuel configuration. From these records, the simplified burnup history shown in Figure 3 was derived to reduce the total number of unique configurations modeled by manual selection of 
burnups that coincide with large changes in the average core power. Although the UWNR occasionally operates at full power for several hours, the present model depletes the core continuously at an average power defined as the difference between successive burnups (selected for the simplified history) divided by the time elapsed between those burnups.

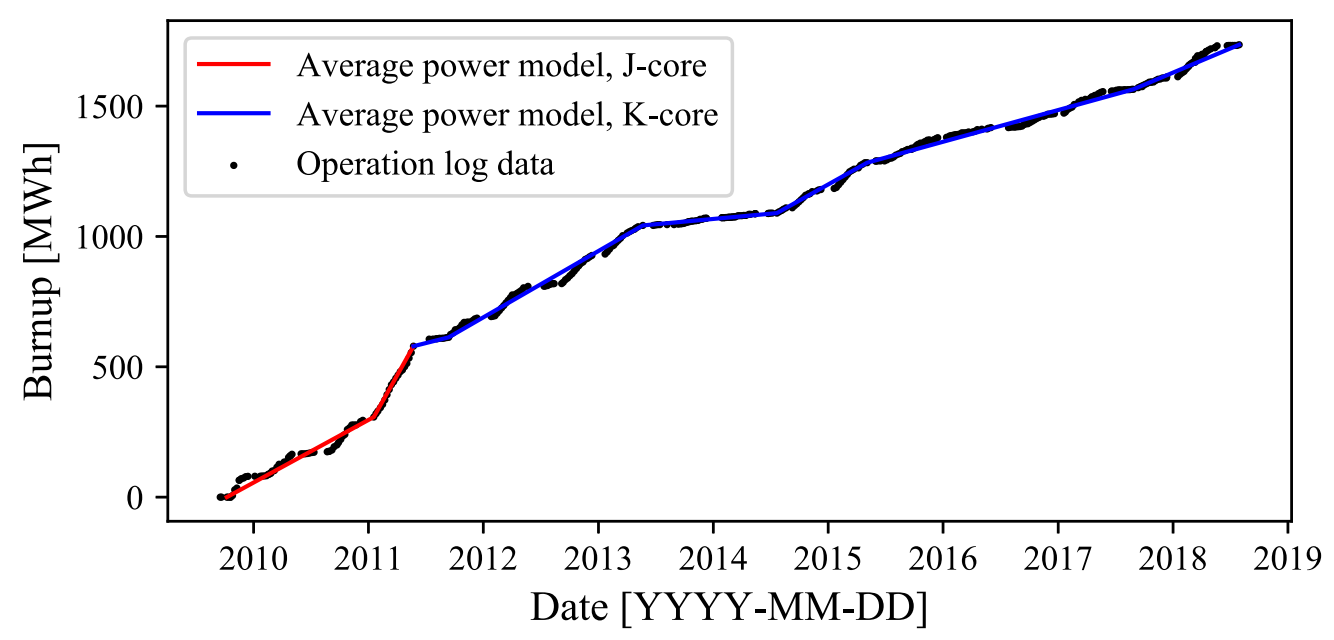

Figure 3: Simplified burnup input model with comparison to operation logs

Preliminary depletion calculations were performed using MCNP6. The core was modeled initially with control elements fully withdrawn in the J-core configuration using slug-averaged, fresh-fuel compositions. A single MCNP6 burnup calculation was performed following the the burnup profile (red) shown in Figure 3. The resulting depleted fuel compositions were parsed and used to define a second, K-core model with all control elements fully withdrawn, which was then depleted following the burnup profile (blue) shown in Figure 3.

For all calculations, ENDF-B/VIII.0 data at $300 \mathrm{~K}$ were used, which represents an approximation to the true thermal conditions. Future analysis will assess the discrepancy introduced by this approximation. For all cases, $10^{5}$ histories per cycle, 20 inactive cycles, and 40 active cycles were used.

Figure 4 shows the predicted and measured cold excess reactivity as a function of core burnup. Solid lines represent original MCNP6 results with 1- $\sigma$ error bars. The bias determined in Section 3.1 can be used to correct for the discrepancy between the simulation results and measurements. Here, a correction of $+1071 \mathrm{pcm}$ from the slug-averaged model was assumed to be constant over the entire fuel burnup and was subtracted from all original MCNP6 results, leading to the biasadjusted results shown as dotted lines.

With the bias adjustment, all predicted reactivities are within 3- $\sigma$ of measured values (for which no formal uncertainty analysis has yet been completed). Moreover, the dependence of reactivity on burnup is consistent between the two data sets. These observations indicate that the newly added core shuffling feature of the UWNR scripted inputs successfully models the continued de- 


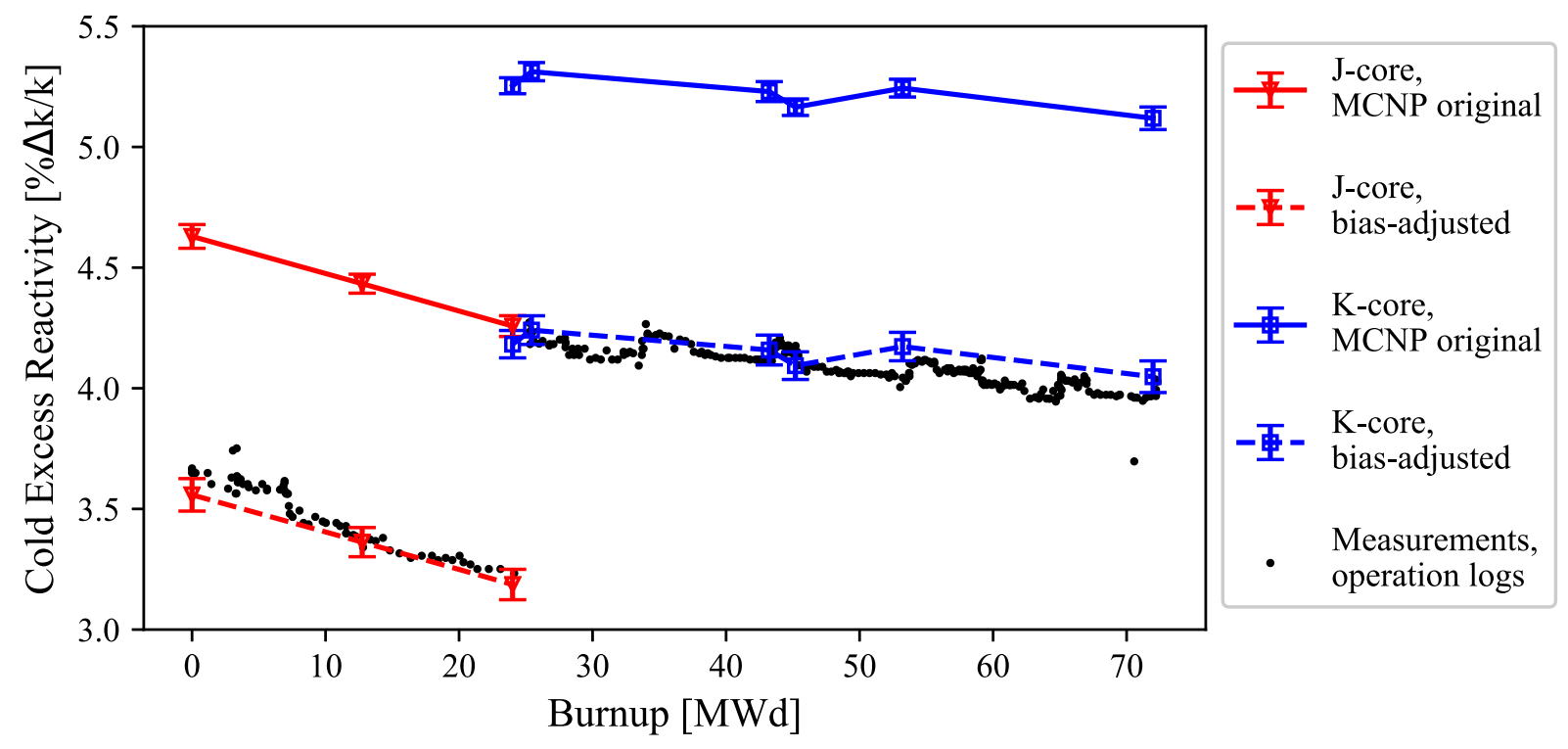

Figure 4: Comparison of cold excess reactivity measurements from operation logs with original and bias-adjusted simulation results by MCNP6

pletion of fuels across different core configurations, and, therefore, provides a starting point for the validation of the UWNR computational model with depleted critical configurations. Future studies will examine the impact of this temperature assumption and identify necessary modeling improvements.

\section{UNCERTAINTY QUANTIFICATION}

\subsection{Nuclear Data Library}

To quantify the uncertainty in the $k_{\text {eff }}$ due to nuclear data uncertainty, the TSUNAMI (Tools for Sensitivity and Uncertainty Analysis Methodology Implementation) module within SCALE code was used. Specifically, continuous energy (CE) TSUNAMI-3D was used with the CLUTCH method[7]. TSUNAMI computes the energy-dependent eigenvalue sensitivity coefficient for each reaction of each isotope in the system, which are then folded with the nuclear data covariance matrix to compute the uncertainty in $k_{\text {eff }}$. These calculations were performed for each ATC configuration using the slug-averaged fuel model. The relative standard deviation of $k_{\text {eff }}$ (i.e, $\left.\frac{\Delta k_{\text {eff }}}{k}\right)$ due to nuclear data uncertainties was found to be in the range of \pm 602 to $625 \mathrm{pcm}$. Also, the computed sensitivity coefficients were inspected, as a function of the ATC configurations, but no obvious trend was observed from which we can deduce sources of bias.

\subsection{Model Geometry}

Preliminary sensitivity analysis on model geometry is presented in this section. A fresh critical J-core configuration with undivided fuel slug geometry and slug-averaged fuel material definitions 
was used as a reference for calculation of uncertainty in $k_{\text {eff. }}$ A number of dimensions from pinlevel to core-level geometries were identified as parameters for perturbation. The uncertainty of each dimension was deduced from currently available drawings based on the number of decimal places provided for the value. Using Python scripts manually modified for direct perturbation, MCNP6 input files were generated with each dimension perturbed by the corresponding uncertainty. For statistics, MCNP6 used $5 \times 10^{6}$ histories per cycle, 20 inactive cycles and 80 active cycles. The eigenvalue result from each MCNP6 run was then subtracted from the eigenvalue of the reference case to represent uncertainty in eigenvalue due to perturbation of corresponding dimension, denoted as $\Delta k_{\text {eff }}$.

Table 1 shows a summary of the perturbed dimensions that resulted in statistically noticeable uncertainties in the eigenvalue. It was found that the eigenvalue is sensitive to small changes in the fuel meat thickness even when the total fuel mass was conserved. Note that significant bias can be introduced even with small modeling error in the cladding thickness. Moreover, it was shown that accurate modeling of the axial positions of the control elements, especially the transient rod and the safety blades, is important. However, the impact of perturbing the axial position of the regulating blade was negligible, as it has relatively small reactivity worth compared to the transient rod and the safety blades.

Table 1: Effect of Uncertainty in Model Geometry

\begin{tabular}{|c|c|c|c|}
\hline Parameter Identification & $\begin{array}{c}\text { Parameter Variation } \\
(\mathrm{cm})\end{array}$ & $\begin{array}{c}\text { Relative Parameter } \\
\text { Change }(\%)\end{array}$ & $\begin{array}{c}\Delta k_{\text {eff }} \pm \sigma_{k_{\text {eff }}} \\
(\mathrm{pcm})\end{array}$ \\
\hline Fuel meat thickness $^{*}$ & 0.03 & 2 & $+175 \pm 6$ \\
\hline Clading thickness & 0.008 & 14 & $-377 \pm 6$ \\
\hline Fuel pin pitch & 0.08 & 2 & $+215 \pm 6$ \\
\cline { 2 - 4 } & 0.03 & 0.8 & $+100 \pm 6$ \\
\hline Active fuel region height $^{\dagger}$ & 0.8 & 2 & $-302 \pm 6$ \\
\cline { 2 - 4 } & 0.3 & 0.8 & $-113 \pm 6$ \\
\hline Transient rod axial position $^{\dagger}$ & 0.8 & - & $+94.5 \pm 6$ \\
\hline Safety blade axial position $^{\dagger}$ & 0.8 & - & $+175 \pm 6$ \\
\hline
\end{tabular}

${ }^{*}$ By design, the fuel density changes accordingly in order to conserve the total fuel mass. Perturbation applied to outer radius that induces more changes in fuel volume than perturbation applied to inner radius.

$\dagger$ Perturbation applied to target dimension in both positive and negative directions $(x-\Delta x$, $x+\Delta x)$. Half of the difference between $\Delta k_{\text {eff }}$ values from positive and negative perturbation was presented as the uncertainty in eigenvalue due to perturbation of the dimension. 


\subsection{Material Composition}

In this section, preliminary sensitivity analysis on material composition is presented. The reference configuration and statistics settings (number of histories and active/inactive cycles) are identical to Section 4.2. An uncertainty quantification study on the material properties of the UWNR computational model was conducted in two approaches, fuel material composition and non-fuel material density.

Limited information is available for uncertainties on per-slug measurements in the as-built fuel material compositions. Hence, the uncertainty for each quantity was defined as the standard deviation over all values for that property across all the as-built data. As in Section 4.2, MCNP6 input files were generated with each property universally perturbed by the corresponding uncertainty, with the help of manually modified Python scripts for direct perturbation. Table 2 shows a summary of the uncertainties in the eigenvalue due to perturbing the fuel material compositions. It was shown that the eigenvalue is sensitive to small changes in most of the properties, indicating the importance of accurate fuel material modeling. Especially, small variations in U-235 mass, Er weight percent and $\mathrm{H} / \mathrm{Zr}$ atomic ratio per slug can lead to significant bias in the model. Further sensitivity analysis on material composition will be conducted with depleted critical configurations to better understand the effect of uncertainty in material composition over the lifetime of the reactor.

Table 2: Effect of Uncertainty in Fuel Material Composition

\begin{tabular}{|c|c|c|c|}
\hline $\begin{array}{c}\text { Parameter Identification, } \\
\text { Per-slug Value }\end{array}$ & Parameter Variation & $\begin{array}{c}\text { Relative Parameter } \\
\text { Change }(\%)\end{array}$ & $\begin{array}{c}\Delta k_{\text {eff }} \pm \sigma_{k_{\text {eff }}} \\
(\mathrm{pcm})\end{array}$ \\
\hline U-235 mass & $0.55 \mathrm{~g}$ & 1.11 & $+208.5 \pm 6$ \\
\hline U mass & $2.69 \mathrm{~g}$ & 1.07 & $-140 \pm 6$ \\
\hline Total mass & $2.09 \mathrm{~g}$ & 0.25 & $+45 \pm 6$ \\
\hline C wt. \% & $0.049 \%$ & 14.95 & $-96 \pm 6$ \\
\hline Er wt. \% & $0.064 \%$ & 7.32 & $-876 \pm 6$ \\
\hline H/Zr atomic ratio & 0.02 & 1.26 & $+202.5 \pm 6$ \\
\hline
\end{tabular}

For uncertainty in non-fuel material modeling, mass densities were perturbed, as the UWNR model uses predefined compositions from the compendium of material compositions[8] provided by PyNE. Table 3 shows the uncertainties in eigenvalue due to perturbing the non-fuel material density. Most of the density perturbations resulted in statistically negligible uncertainties in eigenvalue, except graphite. Further investigation is underway in order to better understand the impact of the uncertainty in graphite modeling on the eigenvalue. 
Table 3: Effect of Uncertainty in Non-fuel Material Density

\begin{tabular}{|c|c|c|c|}
\hline Parameter Identification & Parameter Variation & $\begin{array}{c}\text { Relative Parameter } \\
\text { Change }(\%)\end{array}$ & $\begin{array}{c}\Delta k_{\text {eff }} \pm \sigma_{k_{\text {eff }}} \\
(\mathrm{pcm})\end{array}$ \\
\hline Stainless steel & $0.01 \mathrm{~g} / \mathrm{cc}$ & 0.125 & $12 \pm 6$ \\
\hline Zirconium & $0.01 \mathrm{~g} / \mathrm{cc}$ & 0.153 & $8 \pm 6$ \\
\hline Water & $0.000001 \mathrm{~g} / \mathrm{cc}$ & 0.0001 & $17 \pm 6$ \\
\cline { 2 - 4 } & $3^{\circ} \mathrm{C}$ & 0.06 & $13 \pm 6$ \\
\hline Carbon (graphite) & $0.01 \mathrm{~g} / \mathrm{cc}$ & 0.59 & $31.5 \pm 6$ \\
\hline Boron carbide & $0.01 \mathrm{~g} / \mathrm{cc}$ & 0.4 & $14 \pm 6$ \\
\hline Boral & $0.01 \mathrm{~g} / \mathrm{cc}$ & 0.4 & $18 \pm 6$ \\
\hline Aluminum & $0.01 \mathrm{~g} / \mathrm{cc}$ & 0.37 & $18 \pm 6$ \\
\hline
\end{tabular}

\section{CONCLUSIONS}

Recent addition of SCALE/KENO representation to the model was validated with a set of fresh critical configurations from approach-to-critical measurements. It was observed that the modeling results by KENO provide increased confidence in the UWNR computational model, as they lead to smaller variance on averaged eigenvalues when added to MCNP6 and Serpent results. Also, KENO results were observed to be closer to MCNP6 results than Serpent results, suggesting further investigation of the Serpent model.

Initial efforts to validate the UWNR model with depleted critical configurations in the MCNP6 representation against measurements from operation logs have demonstrated that the model can correctly follow the reactivity changes associated with burnup and depletion. It was shown that general trend of reactivity change with burnup simulated by MCNP6 is parallel to the trend recorded in the operation logs. After adjustment of bias established in fresh critical configurations to all stages of simulated burnup, simulated cold excess reactivities were found to be statistically equivalent to the actual measurements on both J-core and K-core. As a result, it was confirmed that the UWNR computational model with the recent addition of core shuffling feature successfully modeled continued depletion of fuel elements after shuffling of core configuration from J-core to K-core. This will lead to an accurate modeling of depleted fuel material inventories as of today.

Moreover, a preliminary uncertainty quantification study was performed. For the nuclear data induced uncertainty, the TSUNAMI-3D sequence in SCALE was used. The relative standard deviation of $k_{\text {eff }}$ was found to be about $\pm 600 \mathrm{pcm}$. For the uncertainties due to model geometry and material composition, direct perturbation calculations were performed using the MCNP6 model in order to investigate the sensitivity of $k_{\text {eff }}$ to changes on dimensions and material properties which could be either due to modelling error or real uncertainty in the parameters. As a result, model parameters having great impact on the simulated eigenvalue were identified. Results show 
that significant bias on the $k_{\text {eff }}$ can be introduced with small modeling error on cladding and fuel meat thicknesses. Also, $k_{\text {eff }}$ of the UWNR model was found to be sensitive to axial position of the transient rod and the safety blades. For fuel material compositions, uncertainties in U-235 mass, Er weight percent and $\mathrm{H} / \mathrm{Zr}$ atomic ratio were found to have the greatest impact on the $k_{\text {eff }}$. Also, uncertainty in the carbon density seems to have the most significant effect among all other perturbed non-fuel material densities. Future work will involve more in-depth uncertainty quantification study where Monte Carlo sampling technique could be used along with SCALE S/U tools, to identify the major sources of bias.

\section{ACKNOWLEDGEMENTS}

This material is based upon work supported by the DOE Office of Nuclear Energy's Nuclear Energy University Program under Award Numbers DE-NE0008408 and DE-NE0008750. This research was performed using the compute resources and assistance of the UW-Madison Center For High Throughput Computing (CHTC) in the Department of Computer Sciences. The CHTC is supported by UW-Madison, the Advanced Computing Initiative, the Wisconsin Alumni Research Foundation, the Wisconsin Institutes for Discovery, and the National Science Foundation, and is an active member of the Open Science Grid, which is supported by the National Science Foundation and the U.S. Department of Energy's Office of Science. In addition, some of the computing for this project was performed on the Beocat Research Cluster at K-State, which is funded in part by NSF grants CNS-1006860, EPS-1006860, EPS-0919443, ACI-1440548, CHE-1726332, and NIH P20GM113109.

\section{REFERENCES}

[1] Y.-H. Park, A. Swenson, and P. P. Wilson. "University of Wisconsin Nuclear Reactor Modeling Improvements." In Transactions of the American Nuclear Society, volume 116, pp. 10821085. Pennsylvania, US (2017).

[2] Y.-H. Park, A. Swenson, P. P. Wilson, Y. Cheng, R. L. Reed, and J. A. Roberts. "IMPROVED MODELING OF THE UNIVERSITY OF WISCONSIN NUCLEAR REACTOR BY AUTOMATIC GENERATION OF COMPUTATIONAL MODELS." In Proceedings of the PHYSOR 2018, volume 1, pp. 2734-2747. Cancun, Mexico (2018).

[3] J. Leppänen, M. Pusa, T. Viitanen, V. Valtavirta, and T. Kaltiaisenaho. "The Serpent Monte Carlo code: Status, development and applications in 2013." Annals of Nuclear Energy, volume 82, pp. 142-150 (2015).

[4] C. J. Werner(editor). MCNP Users Manual - Code Version 6.2. Los Alamos National Laboratory, New Mexico, US (2017).

[5] Y. Cheng and J. A. Roberts. "A study of reactivity biases and their dependence on spatial discretization in depleted TRIGA fuel." Annals of Nuclear Energy, volume 108, pp. 126-131 (2017).

[6] B. T. Rearden and M. A. Jessee (Eds.). SCALE Code System. Oak Ridge National Laboratory, Tennessee, US (2016).

[7] C. M. Perfetti and B. T. Rearden. "SCALE 6.2 Continuous-Energy TSUNAMI-3D Capabilities." (2015).

[8] R. Mcconn, C. Gesh, R. Pagh, R. Rucker, and R. Williams. "Compendium of Material Composition Data for Radiation Transport Modeling." (2011). 